\title{
Recommendations for Improving Adherence to Home-Based Exercise: A Systematic Review
}

\section{Empfehlungen zur Verbesserung der Adhärenz zu Heimübungsprogrammen: Eine Übersichtsarbeit}

Authors

Caroline Bachmann ${ }^{1}$, Peter Oesch ${ }^{2}$, Stefan Bachmann ${ }^{3,4}$

Affiliations

1 Department of Health Science and Technology, ETH Zürich, Zürich, Switzerland

2 Department of Physiotherapy, Kliniken Valens, Valens, Switzerland

3 Department of Rheumatology, Kliniken Valens, Valens, Switzerland

4 Department of Geriatrics, Inselspital, Bern University Hospital, University of Bern, Bern, Switzerland

Key words

home-based exercise, adherence, influencing factors, recommendations, review

Schlüsselwörter

Heimübungsprogramme, Adhärenz, Einflussfaktoren, Empfehlungen, Review

received 18.05 .2017

accepted 28.09.2017

Bibliography

DOI https://doi.org/10.1055/s-0043-120527

Published online: 18.12.2017

Phys Med Rehab Kuror 2018; 28: 20-31

(c) Georg Thieme Verlag KG Stuttgart · New York ISSN 0940-6689

Correspondence

PD Dr. med. Stefan Bachmann

Klinik für Rheumatologie und internistische Rehabilitation

Kliniken Valens

Valens $\mathrm{CH}-7317$, Switzerland

stefan.bachmann@kliniken-valens.ch

\section{ABSTRACT}

Purpose The aim of this review is to list the factors that influence adherence to home-based exercise, and to make recommendations about how to improve adherence.

Materials and Methods A literature search was performed in Web of Science Core Collection (Library of ETH Zurich), PubMed and PEDro databases. The following search categories were used: "physiotherapy", "home-based exercises", "adherence", "effectiveness", "motivation” and "barriers".
Results A total of 38 studies were identified and title and abstract analysis was performed. 14 studies were included for further analysis. The factors found to contribute to adherence to home-based exercise programmes are: intensive social support and guidance from a physiotherapist; prescription of a low number of exercises (<4); high levels of self-motivation and self-efficacy; and a lack of psychological influences, such as helplessness, depression and anxiety.

Conclusion Adherence to home-based exercise could be increased relatively simply, through provision of good support of the patient from physiotherapists, instruction of 2 to a maximum of 4 exercises, and by increasing the patients' self-motivation and self-efficacy (Recommendation level B +).

\section{ZUSAMMENFASSUNG}

Fragestellung Das Ziel der Übersichtsarbeit ist eine systematische Auflistung von relevanten Faktoren, die die Adhärenz zu Heimübungsprogrammen beeinflussen können, und aus diesen Resultaten Empfehlungen abzuleiten, wie die Adhärenz gesteigert werden kann.

Material und Methoden Es wurde eine systematische Literaturrecherche in den Datenbanken Web of Science Core Collection (Bibliothek der ETH Zürich), PubMed und PEDro durchgeführt. Die folgenden Suchkategorien wurden verwendet: „physiotherapy“, „home-based exercise“, „adherence“, „effectiveness“, „motivation“ und „barriers“.

Ergebnisse Es konnten 38 Studien identifiziert werden, von denen nach der Titel- und Abstractanalyse noch 14 Arbeiten in die Übersichtsarbeit einbezogen wurden. Die Resultate zeigen, dass vor allem intensive soziale Unterstützung und Führung durch einen Physiotherapeuten, geringe Anzahl instruierter Übungen (<4), hohe Selbstmotivation und Selbstwirksamkeit und nicht vorhanden sein von psychologischen Einflüssen wie Hilfslosigkeit, Depressionen und Angstzustände zur Adhärenz zu einem Heimübungsprogramm beitragen können.

Schlussfolgerung Durch eine gute Begleitung des Patienten durch Physiotherapeuten, durch die Instruktion von 2 - max. 4 Übungen und durch Erhöhung der Selbstmotivation und Selbstwirksamkeit der Patienten kann die Adhärenz zu Heimübungsprogrammen relativ einfach gesteigert werden (Recommendation level $\mathrm{B}+$ ). 


\section{Introduction}

Exercise training is an important therapeutic strategy after injury, surgery or diagnosis of a musculoskeletal disease. It has the potential to improve recovery and prevent a decrease in function [1]. Exercise training is therefore an inherent part of most rehabilitation programmes. The so-called "home-based exercise" (HBE) training programme is a major part of the rehabilitation process. This programme comprises different exercises adapted for each patient, which should be carried out independently during rehabilitation and thereafter at home for at least 3 months. The effectiveness of this specific type of training has been described and proved in many studies [2-7]. O'Reilly et al. [8] conclude that a simple home-based exercise programme to strengthen the quadriceps muscles for patients with osteoarthritis of the knee could significantly improve knee pain and function. In a study comparing a home-based rehabilitation programme for patients with chronic low-back pain with standard physical therapy, Frih et al. [9] found that a home-based exercise programme could reduce the intensity of chronic pain and perceived impairment and improve functional capacity.

Patients' adherence to such programmes is central to the success of the therapy [10]. The World Health Organization (WHO) defines adherence as "the extent to which a person's behaviour - taking medication, following a diet, and/or executing lifestyle changes, corresponds with agreed recommendations from a health care provider. [11]" Regarding home-based exercise programmes, poor adherence to therapy is a major problem. A systemic review by Jack et al. [12] found that $14 \%$ of patients undergoing physiotherapy did not return for follow-up outpatient appointments and suggested that non-adherence with treatment and exercise performance could be as high as $70 \%$. Poor adherence can compromise treatment outcomes and lead to recurrence of symptoms [13].

Sometimes, the patients' behaviour must change in order to obtain a higher level of adherence. Models of health behaviour change postulate a pattern of factors that may improve motivation and eventually lead to sustained change in behaviour [14]. One of the most popular models is the so-called "Health Action Process Approach" (HAPA) model. This model is divided into 2 different phases, the motivational phase and the volitional phase. In the motivational phase, a person develops an intention to act. Risk perception, perceived outcome expectancies and perceived self-efficacy are predictors of intention. Therefore, patients are more adherent if they are aware of the dangers of their current life situation, if they expect positive effects from the exercises, and if they are satisfied that they can influence their own health. After a person develops an inclination towards a particular health behaviour, the "good intention" in the volitional phase has to be transformed into detailed instructions on how to perform the desired action. In this phase, patients are classified into 3 different stages, which differ according to the patients' mental state. These stages are called non-intenders or pre-intenders, intenders and actors. The determination of these stages takes place with the help of a "stage assessment questionnaire" $[14,15]$. Non-intenders are unmotivated and inactive and benefit from a motivational intervention in which the risks and resources are communicated. Intenders are motivated but inactive and require good planning of the desired action and barrier-focused strategic planning to become active. Actors are in the best state to maintain their health behaviour because they are mo- tivated and active. With the help of relapse prevention interventions, good behaviour can be stabilized $[14,15]$.

However according to a study by Palazzo et al. [13] there are many patient-related factors that could lead to termination of the prescribed home-based exercise programme. These factors are: poor self-efficacy; fear of pain; or inability to fit exercises into daily life. Understanding of such barriers may help clinicians to identify patients at risk of non-adherence and suggest methods to reduce the effect of those barriers and thereby to maximize adherence.

The purpose of this systematic review is therefore to determine all relevant factors that could influence adherence to home-based exercise programmes.

\section{Materials and Methods}

\section{Search strategy}

A systematic review of the literature was performed. The following databases were searched: Web of Science Core Collection (Library of ETH Zurich), PubMed and PEDro. Search categories comprised: terms relating to home physical therapies ("physiotherapy", "home exercise program", "home-based program”, "home exercises"), terms concerning adherence ("adherence”, “compliance”, "exercise maintenance"), terms concerning effectiveness of home programmes ("effectiveness", "efficacy”, “effect”), terms concerning patient-related factors ("motivation", "self-motivation", "self-efficacy") and terms referring to predictors ("predictors”, "barriers"). References to primary studies were scanned to identify further relevant citations. Internet searches of Google and Google Scholar were also conducted.

\section{Selection criteria}

All types of studies with a quantitative design were accepted, with the exception of doctoral theses. The age of the patients, the type of illness, and when the study was published were not relevant for this systematic review. No language restrictions were applied. The selected studies were searched for factors that could influence adherence to home-based exercise.

Excluded were qualitative studies and trials that did not investigate adherence to exercise programmes, investigated the adherence to exercise delivered via new technology and investigations regarding medication-related adherence.

One reviewer (CB) screened titles, abstracts and full texts. Disagreements were resolved through discussions involving the other 2 researchers $(\mathrm{SB}, \mathrm{PO})$.

\section{Quality assessment}

Study evidence levels (and therefore study quality) were assessed according to the Oxford Centre for Evidence-Based Medicine (OCEBM) levels of evidence [16] ( Table 1). Recommendations were also based on the OCEBM system and were rated as follows: $(+)$ = positive correlation; $(-)=$ negative correlation with adherence to home-based exercise.

\section{Data synthesis}

Due to a lack of homogeneity across studies a meta-analytic approach to synthesis was not possible. Therefore, no statistical analyses or meta-analysis were performed. 


\section{Results}

\section{Study flow}

38 potentially relevant publications were identified. Of these, 18 were excluded after reading the abstract because they had no relation to adherence, compliance or exercise maintenance. A further 6 articles were excluded due to the selection criteria; thus a final total of 14 papers were analysed further. Details are shown in $>$ Fig. 1.

\section{Description of studies}

Of the 14 included studies, 4 were systematic reviews, 2 were randomized controlled trials of low quality, 7 were prospective studies, and one was a cross-sectional study ( $\triangleright$ Table 2). 6 studies were published in North America (USA), one in South America (Columbia), 2 in Europe (Spain and Netherland), one in Australia, and the 4 reviews were published in the United Kingdom.

- Table 1 Oxford Centre for Evidence-Based Medicine (OCEBM) levels of evidence [16].

\begin{tabular}{|c|c|c|}
\hline Recommendations & Evidence level & Description \\
\hline \multirow[t]{3}{*}{ A } & $1 \mathrm{a}$ & Systematic reviews (with homogeneity) of randomized controlled trials \\
\hline & $1 \mathrm{~b}$ & Individual randomized controlled trials (with narrow confidence interval) \\
\hline & $1 c$ & $\begin{array}{l}\text { All or none randomized controlled trials (all patients die before the application of treatment, and some } \\
\text { patients survive after treatment; or some patients die before the application of treatment and no patient } \\
\text { dies after treatment) }\end{array}$ \\
\hline \multirow[t]{4}{*}{ B } & $2 a$ & Systematic reviews (with homogeneity) of cohort studies \\
\hline & $2 b$ & Individual cohort study or low quality randomized controlled trials (e. g. <80\% follow-up) \\
\hline & $3 a$ & Systematic reviews (with homogeneity) of case-control studies \\
\hline & $3 b$ & Individual case-control study \\
\hline $\mathrm{C}$ & 4 & Case-series (and poor quality cohort and case-control studies) \\
\hline $\mathrm{D}$ & 5 & Expert opinion or comment \\
\hline
\end{tabular}

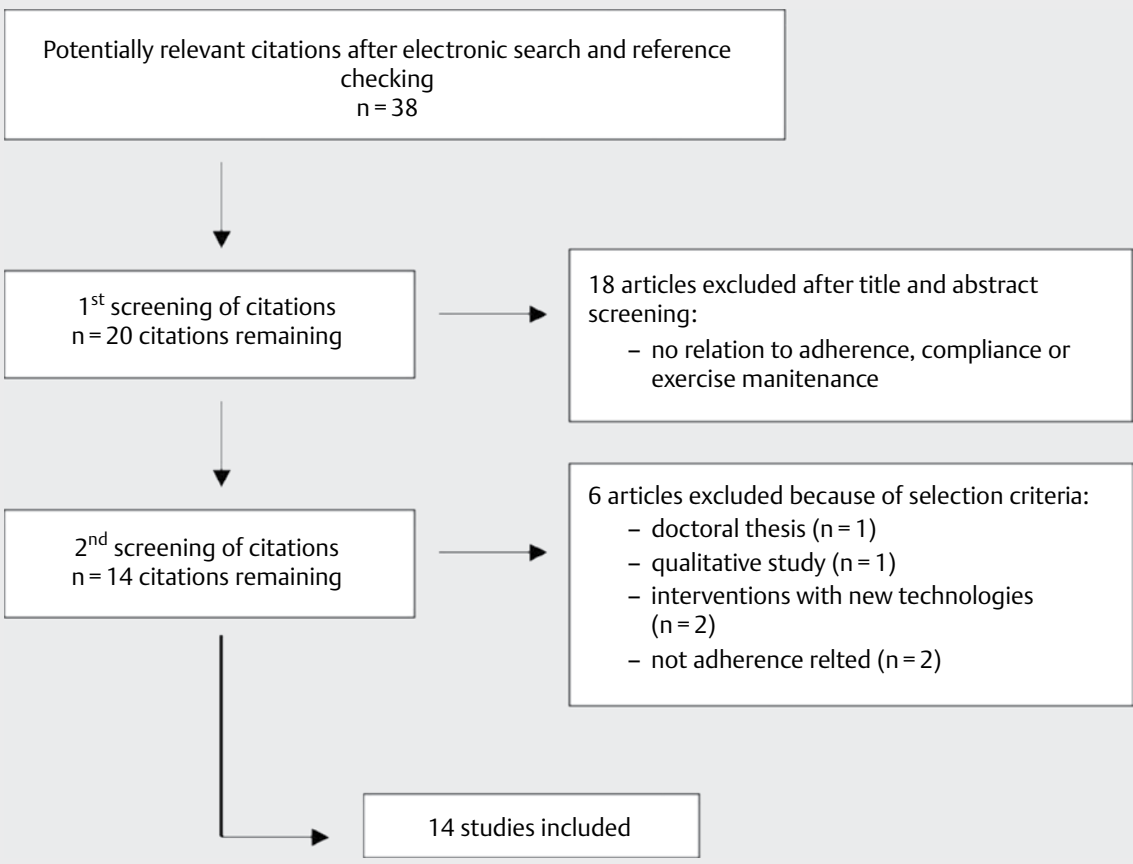

- Fig. 1 Study flow diagram outlining the search process. 


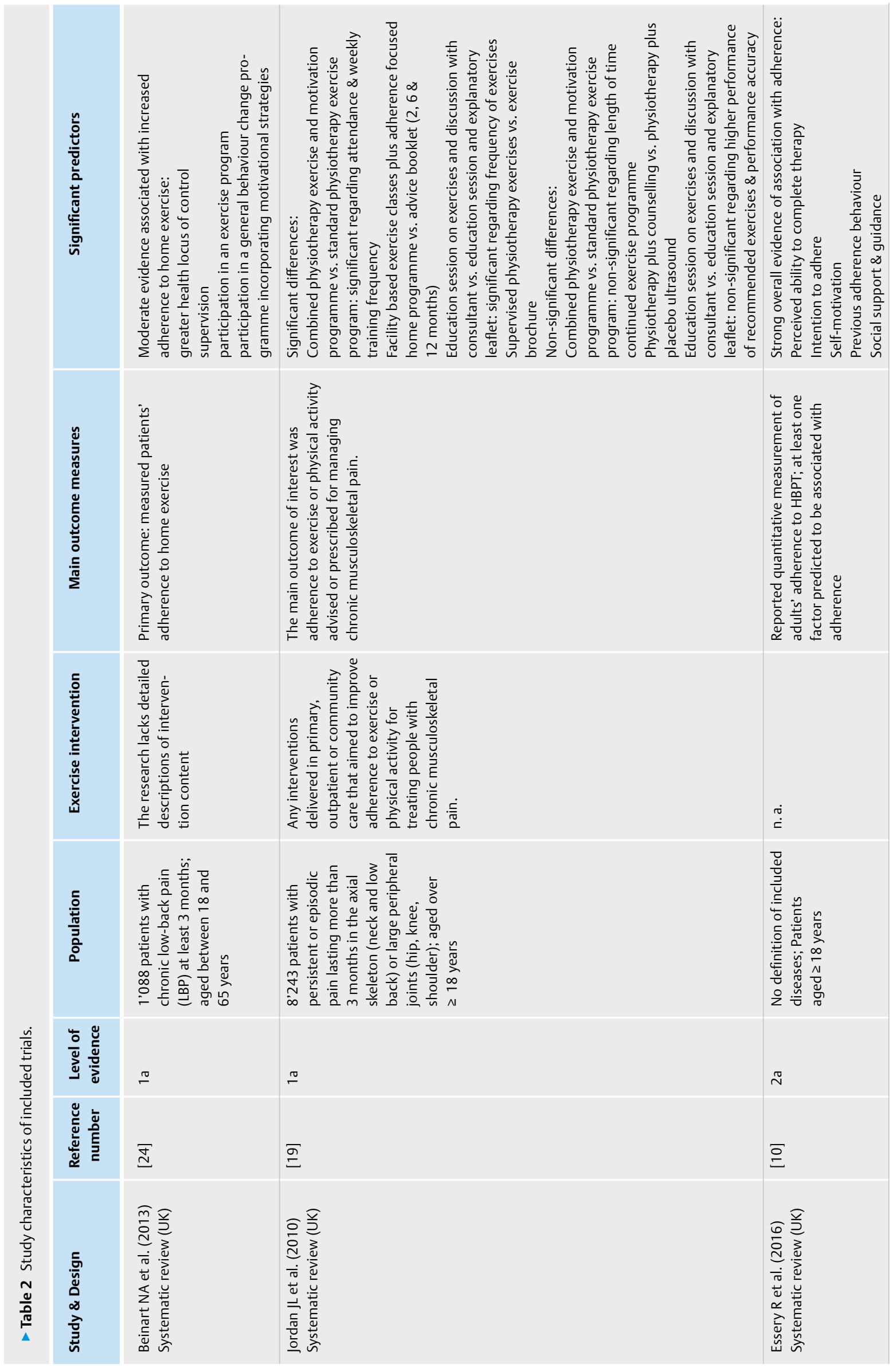




\begin{tabular}{|c|c|c|c|c|}
\hline 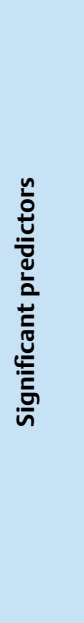 & 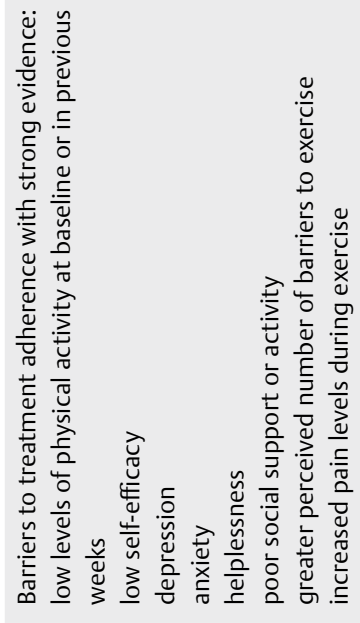 & 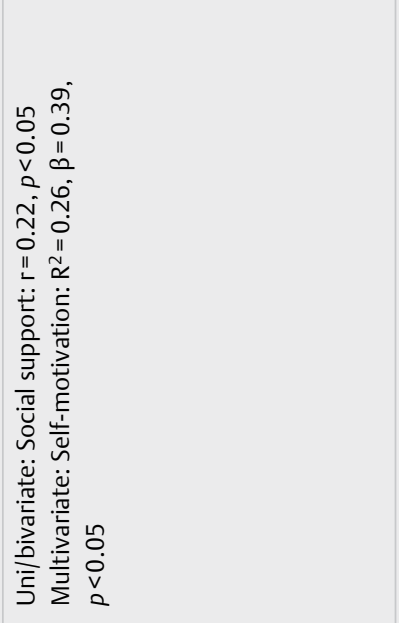 & 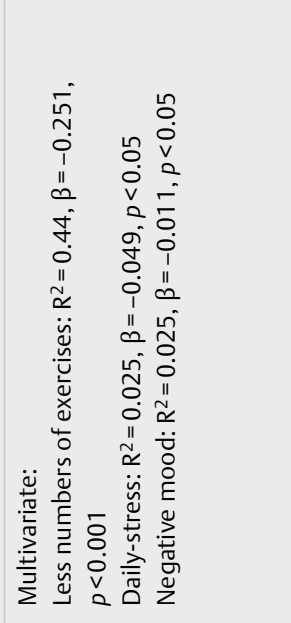 & 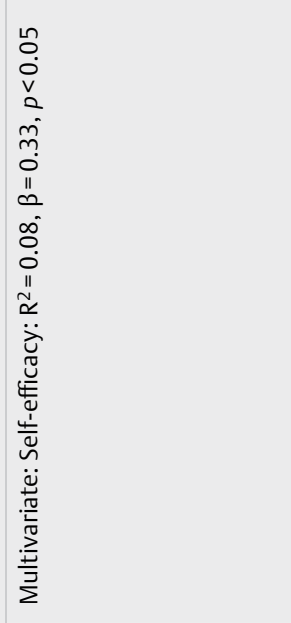 \\
\hline 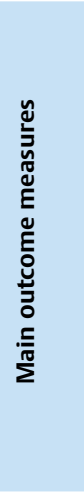 & 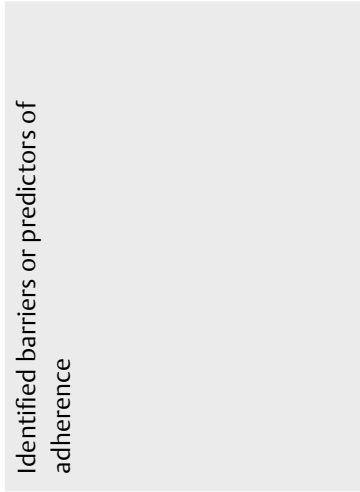 & 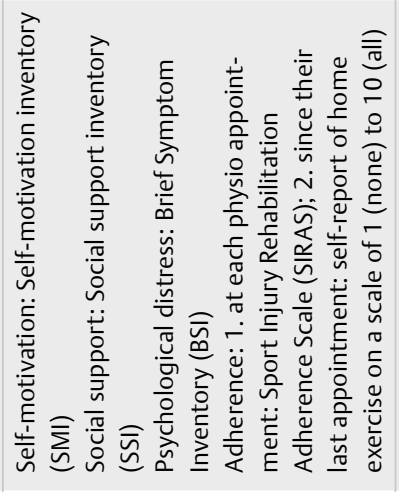 & 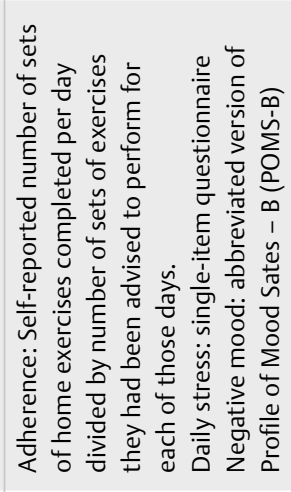 & 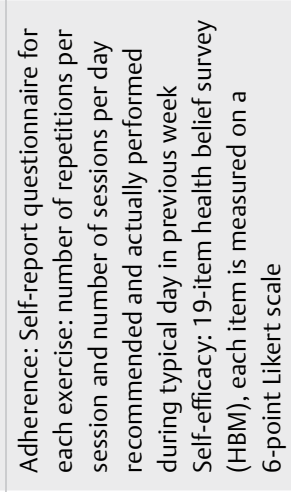 \\
\hline 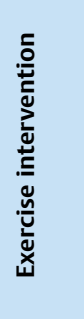 & 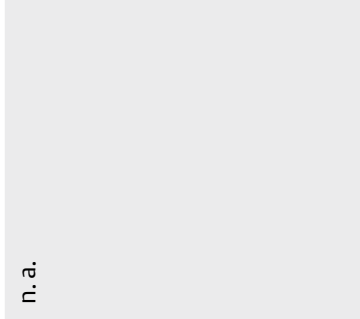 & 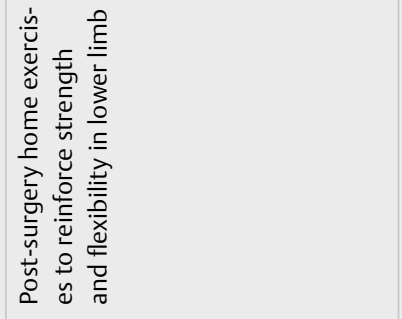 & 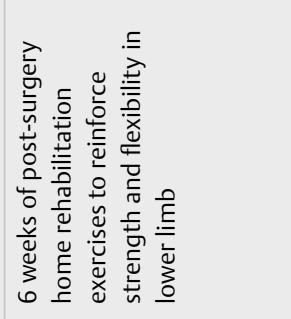 & 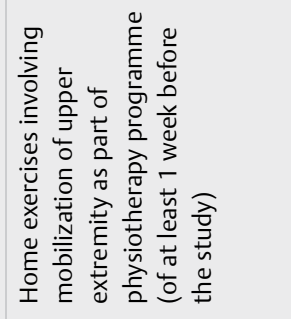 \\
\hline 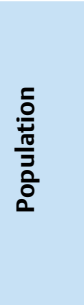 & 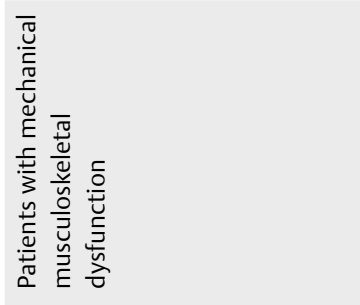 & 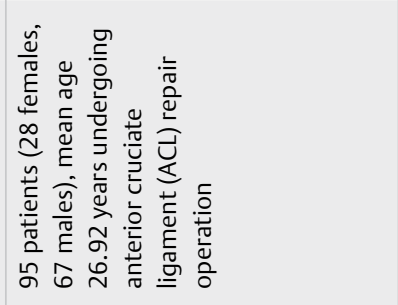 & 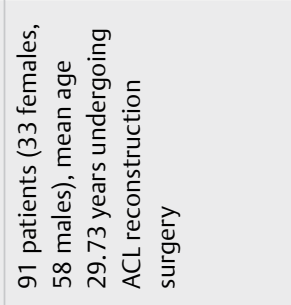 & 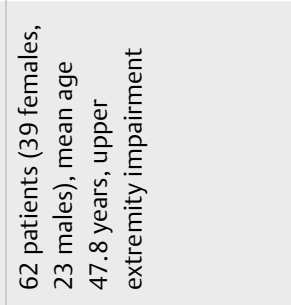 \\
\hline 힘 & $\widetilde{\sim}$ & $\stackrel{\sim}{\sim}$ & $\hat{\sim}$ & $\stackrel{\vec{N}}{ }$ \\
\hline 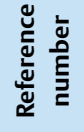 & $\underset{\Xi}{\Xi}$ & $\overline{\underline{\beth}}$ & 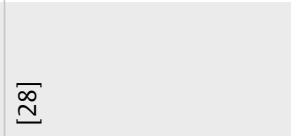 & $\stackrel{\bar{\coprod}}{\varrho}$ \\
\hline 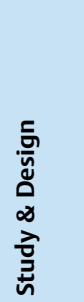 & 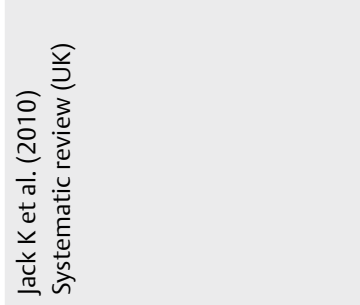 & 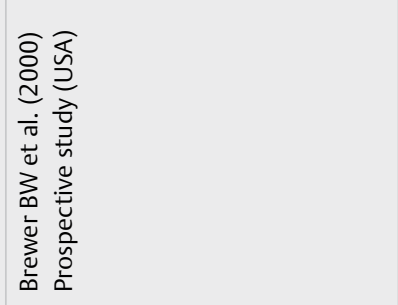 & 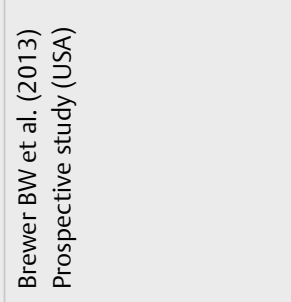 & 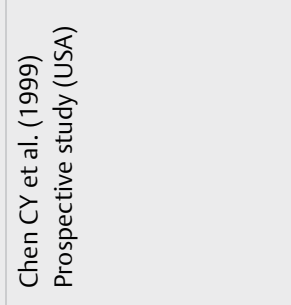 \\
\hline
\end{tabular}




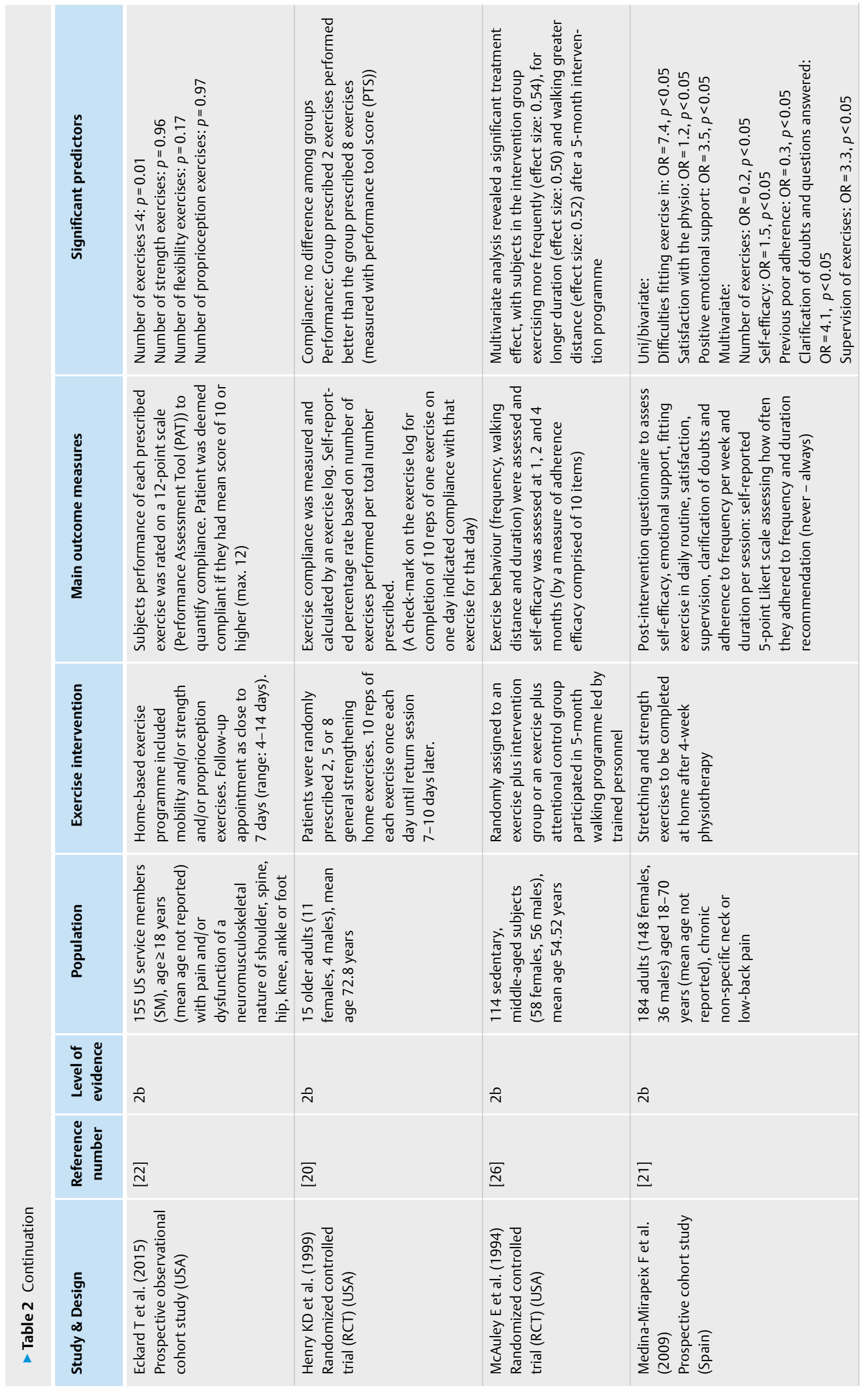



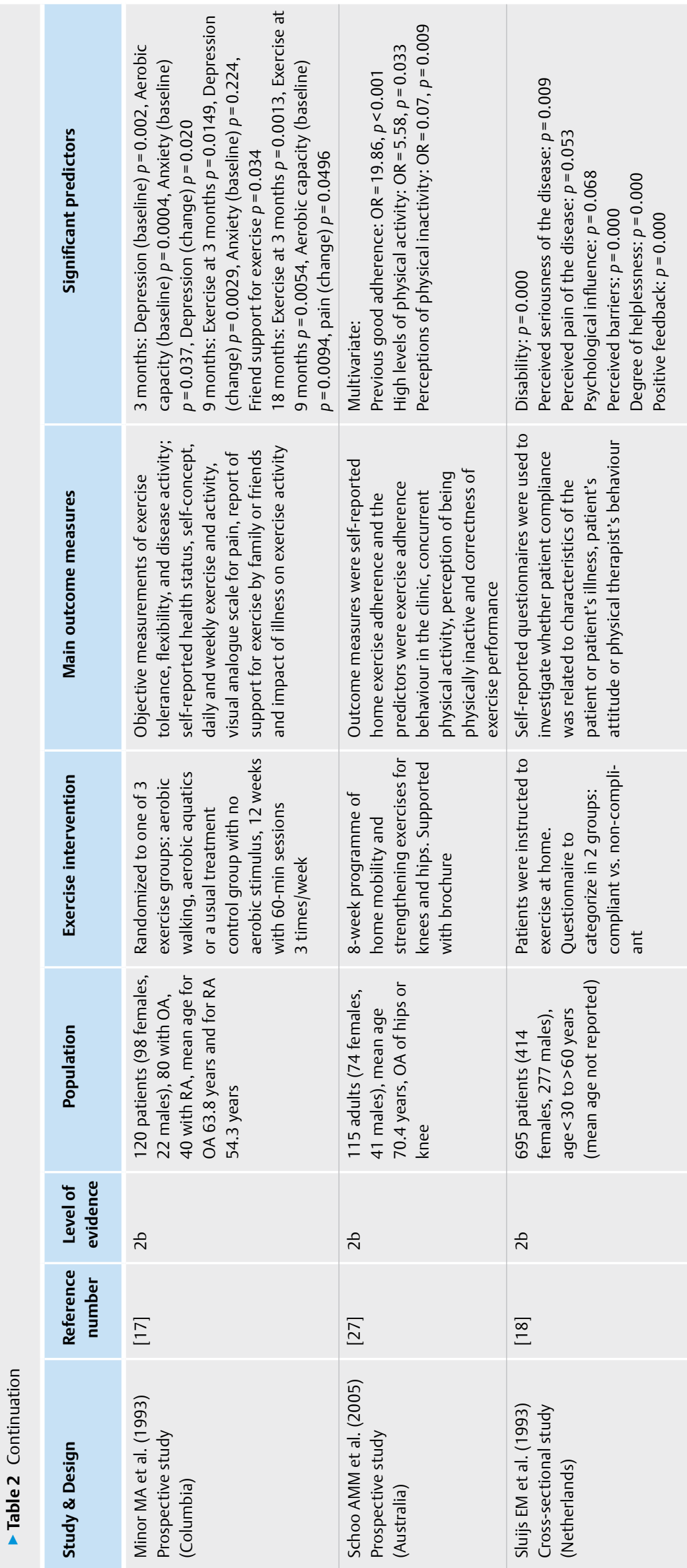


\section{Adherence measures}

Adherence was mostly measured via self-reported questionnaires or logbooks, according to the number of exercises completed divided by the number of exercises prescribed.

\section{Factors associated with adherence to home-based exercise}

The following 10 items were found to be important for patients' adherence: social support, guidance, number of exercises, self-motivation, self-efficacy, previous adherence behaviour, low level of physical activity or aerobic capacity at baseline, exercise attention, worsening of pain during exercises, and high degree of helplessness, depression and anxiety (for details see $>$ Table 2 ).

\section{1) Social support}

3 studies $[10,12,17]$ concluded that low levels of social or familial support were barriers to adherence to treatment. In patients with osteoarthritis (OA) or rheumatoid arthritis (RA) [17], the effect of having support with exercise positively predicted exercise behaviour 9 months after participating in an exercise class. Emotional support and listening support from team-mates and personal assistance from family were associated with greater adherence to home-based exercise [10]. This is a good way of providing some patients with the social stimulation and long-term encouragement to continue their exercise progression [12].

\section{2) Guidance}

4 studies $[10,12,18,19]$ investigated the relationship between guidance from a physiotherapist and adherence to home-based exercise. In subjects with musculoskeletal pain, lack of positive feedback from a physical therapist predicted non-adherence with a home exercise programme prescribed as part of physical therapy rehabilitation [18]. Support provided by the physiotherapist, development of the patient-practitioner relationship, positive feedback from the physiotherapist, follow-up to reinforce exercise behaviour and supplementing face-to-face instruction with other material could have a positive influence on levels of adherence to exercise $[12,19]$.

\section{3) Number of exercises}

The number of exercises prescribed by a home-based exercise programme was investigated by 3 studies [20-22]. 2 studies found that if more than 4 exercises were prescribed patients were less likely to adhere to recommendations [21,22]. 2 studies found that patients are more adherent if 3 or fewer exercises are prescribed $[20,21]$. These results suggest that prescribing a higher number of therapeutic exercises may not always benefit the patient [22]. However, according to the study by Henry et al. [20], further research is needed into the optimal number of exercises to prescribe.

\section{4) Self-motivation}

3 studies investigated the effect of patients' self-motivation to engage in home-based exercise $[10,23,24]$. One study demonstrated that lower self-motivation (measured with the Self-Motivation Inventory (SMI)) contributed to decreased adherence to a homebased exercise programme [23]. Weekly training frequency in those with high motivation was significantly greater than in those with low motivation [24].

\section{5) Self-efficacy}

Low self-efficacy was identified as a barrier to treatment adherence $[12,25,26]$. In subjects attending an upper limb rehabilitation centre, greater self-efficacy (measured by a 19-item health belief survey (HBM)) contributed to greater adherence to home-based exercise [25]. One study demonstrated that patients with greater self-efficacy tend to be more adherent to outpatient physical therapy [12] and, therefore, interventions to increase self-efficacy can effectively reduce non-adherence to exercise programmes [26].

\section{6) Previous adherence behaviour}

A systematic review by Essery et al. [10] provided evidence that patients who had previously demonstrated good adherence to home-based exercise, or who had not demonstrated poor adherence were more likely to be adherent at the current time point.

\section{7) Low level of physical activity or aerobic capacity at baseline}

2 studies found that patients undergoing home-based exercise with OA or RA [17, 27] were more likely to adhere to a home-based exercise programme if they were physically active at baseline or had a high aerobic capacity at baseline.

\section{8) Exercise attention}

Possible barriers to exercise include not having time for exercise, inability to fit exercises into daily life, and forgetting to do exercises.

In patients with musculoskeletal pain, the barriers patients perceived and encountered were: lack of time, lack of convenience, costs, and forgetting to do exercises. These factors predicted non-adherence with a home-based exercise programme prescribed during physical therapy rehabilitation [18].

\section{9) Worsening of pain during exercises}

Worsening of pain during exercise was a barrier to adherence to home-based exercise, because one study [17] confirmed that improvement in pain following participation in an exercise class positively predicted exercise behaviour.

\section{0) High degree of helplessness, depression and anxiety}

Depression, anxiety and helplessness were also barriers to treatment adherence $[12,17,18]$. One study [17] confirms that depression could influence patients' adherence to home-based exercise programmes. Less depression (measured by the Arthritis Impact Measurement Scale (AIMS)) in patients with OA/RA [17] predicted exercise maintenance 3 months after participating in an exercise programme. Furthermore, patients with musculoskeletal pain who had a greater feeling of helplessness (measured by a 12-item scale scored on a 4-point scale varying from 1 ("agree") to 4 ("disagree")) were predicted to be non-adherent with a home-based exercise programme prescribed as part of physical therapy rehabilitation [18]. Participants completed fewer home exercises on days when they experienced more stress (measured by a single item ("How stressful was your day?") scored on a scale from 1 ("not") to 5 ("very")) or negative mood (measured with an abbreviated version of the Profile of Mood States - B (POMS-B)) [28]. 
- Table 3 Nature of effect and levels of evidence according to Oxford Centre for Evidence-Based Medicine (OCEBM).

\begin{tabular}{|c|c|c|c|c|c|c|c|c|c|c|c|c|}
\hline & \multicolumn{12}{|c|}{ Levels of evidence } \\
\hline & \multicolumn{3}{|c|}{$1 a$} & \multicolumn{3}{|c|}{$1 b$} & \multicolumn{3}{|c|}{$2 a$} & \multicolumn{3}{|c|}{$2 b$} \\
\hline & + & - & n.a. & + & - & n.a. & + & - & n.a. & + & - & n.a. \\
\hline Existence of social support & & & & & & & {$[10,12]$} & & & [17] & & \\
\hline $\begin{array}{l}\text { Existence of guidance } \\
\text { through a physiotherapist }\end{array}$ & [19] & & & & & & {$[10,12]$} & & & [18] & & \\
\hline $\begin{array}{l}\text { High number of exercises } \\
\text { prescribed }\end{array}$ & & & & & & & & & & & [20-22] & \\
\hline $\begin{array}{l}\text { Existence of low self-motiva- } \\
\text { tion }\end{array}$ & & {$[24]$} & & & & & & {$[10]$} & & & [23] & \\
\hline Existence of low self-efficacy & & & & & & & & {$[12]$} & & & {$[25-26]$} & \\
\hline $\begin{array}{l}\text { High previous adherence } \\
\text { behaviour }\end{array}$ & & & & & & & {$[10]$} & & & & & \\
\hline $\begin{array}{l}\text { Low level of physical activity } \\
\text { or aerobic capacity at } \\
\text { baseline }\end{array}$ & & & & & & & & & & & {$[17,27]$} & \\
\hline $\begin{array}{l}\text { Exercise attention: not } \\
\text { having time for exercises; } \\
\text { inability to fit exercises into } \\
\text { daily life; forgetting to do } \\
\text { exercises }\end{array}$ & & & & & & & & & & & {$[18]$} & \\
\hline $\begin{array}{l}\text { Worsening of pain during } \\
\text { exercises }\end{array}$ & & & & & & & & & & & [17] & \\
\hline $\begin{array}{l}\text { Existence of high degree of } \\
\text { helplessness, depression or } \\
\text { anxiety }\end{array}$ & & & & & & & & {$[12]$} & & & {$[17,18,28]$} & \\
\hline
\end{tabular}

- Table 4 Summary of recommendation levels according to Oxford Centre for Evidence-Based Medicine (OCEBM).

\section{Adherence influencing factors}

Recommendation level *

Existence of social support

Existence of guidance through a physiotherapist

High number $(>4)$ of exercises prescribed

$B+$

A to $B+$

$B$ -

A to $B$ -

Existence of low self-motivation

Existence of low self-efficacy

High previous adherence behaviour

$B-$

Low level of physical activity or aerobic capacity at baseline

Appearance of barriers to exercise: not having time for exercises; inability to fit exercises into daily life; forgetting to do exercises

Worsening of pain during exercises

Existence of high degree of helplessness, depression or anxiety

${ }^{*}(+)$ positive correlation with adherence to home-based exercise; $(-)$ negative effect on adherence to home-based exercise 


\section{Recommendations}

Based on study evidence levels ( $\vee$ Table 3 ) and according to OCEBM recommendation levels, physiotherapists should guide patients narrowly (Recommendation level $\mathrm{A}-\mathrm{B}+$ ) and it is recommended that social support is provided for patients while they are exercising (Recommendation level $\mathrm{B}+$ ).

Further knowledge of previous high adherence behaviour (as a personal factor of the patients), is also positively correlated with adherence to home-based exercise (Recommendation level B+). All other factors, especially high number of exercises prescribed, are negatively correlated with adherence to home-based exercise ( Table 4).

\section{Discussion}

\section{Summary of key findings}

This systematic review summarized the results from 14 studies and found that poor social support and guidance, high number of exercises, low self-motivation and self-efficacy and psychological influences, such as helplessness, depression and anxiety, are key barriers to patients' adherence to home-based exercise.

\section{Comparison with other studies}

The results of this review show that social support from family members or friends is key to improving adherence to home-based exercise. Social support was also found to play a major role in treatment adherence in patients regarding medication treatments for chronic diseases. For example, patients with diabetes have to be on lifelong medication, and thus a high degree of adherence to such treatment methods is required to achieve a complaint-free life. A study by Garay-Sevilla et al. [29] found that social support appeared to be the main determinant of compliance with medication. This finding is in agreement with the results of our analysis, in which social support seems to be a strong predictor of adherence to home-based exercise.

Furthermore, according to another study of patients with type 2 diabetes by Jeragh-Alhaddad et al. [30], families were frequently quoted as an important source of support, providing moral as well as practical assistance to participants. The patients reported that their family members often reminded them to take their medication or to perform self-monitoring activities.

Nevertheless, further results [29] showed that patients with rigid control in their families had lower scores regarding adherence to medication. Rigid control may enhance the development of conflict with authority, and increase patient's denial of the disease. The result of such denial is decreased compliance with treatment. These findings suggest that social support is important for maintaining the treatment, and that partners should be involved in the rehabilitation process in order to encourage and motivate patients; however, the support should not be so rigid as to have a contrary effect.

Our analysis reveals that the lower the number of prescribed exercises, the higher the adherence rate. The optimum number of exercises is between 2 and 4 in an individual, tailored home-based exercise programme. If patients want to vary their practice, the physiotherapist could instruct more than 4 exercises. This finding is also confirmed in a review from Osterberg et al. [31], which investigated adherence to medication. They found that adherence was inversely proportional to the frequency of intake of medication. This means that simple dosing (one pill, once daily) helps to maximize adherence and, in conclusion, the lower the medication schedule, the higher the expected adherence rate.

A recently published study by Schäfer [32] examined barriers and factors supporting home-based exercise in patients with chronic low-back pain. The author found that, from the patients' perspective, the main barriers were poor motivation, lack of social support, pain during exercise, and lack of time due to work. Furthermore, the main supporting factors included increased motivation by physical therapy, social support or individual goals, education, and provision of exercise sheets. These results are consistent with our findings, although Schäfer included only patients with chronic low-back pain and our analysis included any type of musculoskeletal complaints.

\section{Differences between countries}

It is important to examine the effect of different healthcare systems in different continents or countries. Factors such as health insurance companies and insured benefits may vary among patients. For example, based on the study by Osterberg (USA) et al. [31] patients' non-adherence to pharmacological treatments could occur due to the high cost of medications. In Switzerland, the health insurance companies accept the costs of medication therapy and should therefore not be a reason for non-compliance with treatment. If we adapt this fact to our analysis, it might be possible that, for example, further support from the physiotherapist or other face-to-face instructions to improve adherence are additional services and are not covered by health insurance in some countries and, thus, patients may forgo this service due to the higher personal costs. These obstacles should be circumvented by physiotherapists checking patients' health insurance companies at the first meeting.

Comparisons of the significant predictor "number of exercises", by Medina et al. (Spain) [21] and Brewer et al. (USA) [28] concluded that the prescribed number of exercises could influence patients' adherence to home-based exercise. Medina et al. [21] focused on patients with low-back pain, whereas Brewer et al. [28] only included patients undergoing $A C L$ reconstruction surgery. The same observation is seen in studies of the significant predictor "self-efficacy". Authors from both the USA [25] and Spain [21] found that low self-efficacy could affect adherence to home exercises. Thus, there are no differences in the key findings regarding factors influencing adherence in studies from different continents.

\section{Strengths and limitations}

\section{Strengths}

All types of published articles were included in this systematic review and, although there was no age limit, all participants in the 14 included studies were over 18 years of age. Since all types of articles were included, results from different countries and continents could be analysed and compared. This review therefore provides a good worldwide picture of current knowledge on this topic.

\section{Limitations}

Given the limited number of studies included, some factors influencing adherence, which might be of importance for the physio- 
therapists or rehabilitation teams, may have been missed. Although we applied no language restrictions in study selection, we may have missed studies because we only used English search terms. As qualitative studies cannot be rated according to the OCEBM levels of evidence, and therefore no recommendations can be formulated, these papers were not included in this review. Of the 14 studies included, only 2 are randomized controlled trials (RCT). RCT are referred to as the gold standard for research, and reviews are therefore evaluated according to the number of RCTs included. However, for the topic studied here: "Which factors influence adherence to home-based exercise?", RCTs are not the best study design, because it is not necessary to compare an intervention group with a control group in order to determine factors influencing adherence in physiotherapy settings. Thus, it makes sense to include designs other than RCTs.

The most frequently reported diseases in the included studies are chronic (non-specific) low-back pain and osteoarthritis. Since these patients benefit from regular physical activity, these 2 diseases were most frequently included in the studies. However, it would be of interest to determine whether the factors found to influence adherence also play a major role in diseases in which physical activity is not a leading treatment strategy; for example, all types of cancer. After a cancer diagnosis, surgery, radiation or chemotherapy are the first-line treatments, perhaps followed by physiotherapy depending on cancer type. Physical activity can minimize cancer-related side-effects, such as fatigue or lymphomatous swelling, which can occur due to lymph node excision. In order to gain more insight into factors influencing adherence to $\mathrm{HBE}$, a wide range of diseases as well as patients with different levels of functioning and participation should be included in further research protocols. It can be assumed that in patients with severe restrictions in functioning and participation adherence to HBE is influenced by other factors. During this systematic review we did not find any literature covering this important topic.

A further limitation could be that the publication date of the included studies was not limited; the publication dates range from 1993 to 2016 . Some of these studies may be outdated and research may have advanced. However, for our research question, the publication date is not relevent, since any factors influencing adherence to home-based exercise are important.

\section{Conclusion}

This systematic review identified 14 studies investigating factors that predicted non-adherence to home-based exercise programmes. It appears that poor social support from family members or friends, poor guidance from physiotherapists, greater numbers of prescribed exercises, low self-motivation and self-efficacy are all barriers to adherence to home-based exercise. Identification and overcoming of these barriers may be important in order to ensure an improvement in patients' impairments during the whole rehabilitation process. The results of this systematic review suggest that physiotherapists should be informed about the problems and barriers affecting their patients. With this information, physiotherapists could find solutions to manage the relevant problems and thus, adherence to homebased exercise could be increased relatively simply.

\section{Conflict of Interest}

The authors declare that they have no conflict of interest.

Literatur

[1] Yu-Yahiro JA, Resnick B, Orwig D et al. Design and implementation of a home-based exercise program post-hip fracture: the Baltimore hip studies experience. PM \& R: the journal of injury, function, and rehabilitation 2009; 1: 308-318

[2] Ashari A, Hamid TA, Hussain MR et al. Effectiveness of individualized home-based exercise on turning and balance performance among adults older than 50 yrs: a randomized controlled trial. Am J Phys Med Rehab 2016; 95: 355-365

[3] Anar SO. The effectiveness of home-based exercise programs for low back pain patients. J Phys Ther Sci 2016; 28: 2727-2730

[4] Hill KD, Hunter SW, Batchelor FA et al. Individualized home-based exercise programs for older people to reduce falls and improve physical performance: a systematic review and meta-analysis. Maturitas 2015; 82: 72-84

[5] Thomas KS, Muir KR, Doherty M et al. Home based exercise programme for knee pain and knee osteoarthritis: randomised controlled trial. BMJ (Clinical research ed) 2002; 325: 752

[6] Latham NK, Harris BA, Bean JF et al. Effect of a home-based exercise program on functional recovery following rehabilitation after hip fracture: a randomized clinical trial. JAMA 2014; 311: 700-708

[7] Anwer S, Alghadir A, Brismee JM. Effect of home exercise program in patients with knee osteoarthritis: a systematic review and meta-analysis. Journal of Geriatric Physical Therapy (2001) 2016; 39: 38-48

[8] O'Reilly SC, Muir KR, Doherty M. Effectiveness of home exercise on pain and disability from osteoarthritis of the knee: a randomised controlled trial. Annals of the Rheumatic Diseases 1999; 58: 15-19

[9] Frih ZBS, Fendri Y, Jellad A et al. Efficacy and treatment compliance of a home-based rehabilitation programme for chronic low back pain: a randomized, controlled study. Ann Phys Rehabil Med 2009; 52: 485-496

[10] Essery R, Geraghty AW, Kirby S et al. Predictors of adherence to home-based physical therapies: a systematic review. Disabil Rehabil 2016, doi:10.3109/09638288.2016.1153160: 1-16

[11] Sabaté E. Adherence to long-term therapies: evidence for action. World Health Organization; 2003

[12] Jack K, McLean SM, Moffett JK et al. Barriers to treatment adherence in physiotherapy outpatient clinics: a systematic review. Man Ther 2010; 15: $220-228$

[13] Palazzo C, Klinger E, Dorner V et al. Barriers to home-based exercise program adherence with chronic low back pain: patient expectations regarding new technologies. Ann Phys Rehabil Med 2016; 59: 107-113

[14] Schwarzer R. Modeling health behavior change: how to predict and modify the adoption and maintenance of health behaviors. Appl Psychol-Int Rev 2008; 57: 1-29

[15] Schwarzer R, Lippke S, Luszczynska A. Mechanisms of health behavior change in persons with chronic illness or disability: the Health Action Process Approach (HAPA). Rehabil Psychol 2011; 56: 161-170

[16] Howick J, Chalmers I, Glasziou P et al. The Oxford Levels of Evidence 2. In, Oxford Centre for Evidence-Based Medicine

[17] Minor MA, Brown JD. Exercise maintenance of persons with arthritis after participation in a class experience. Health Education Quarterly 1993; 20: 83-95

[18] Sluijs EM, Kok G], van der Zee J. Correlates of exercise compliance in physical therapy. Physical Therapy 1993; 73: 771-782 
[19] Jordan JL, Holden MA, Mason EE et al. Interventions to improve adherence to exercise for chronic musculoskeletal pain in adults. Cochrane Database Syst Rev 2010, doi:10.1002/14651858. CD005956.pub2: CD005956

[20] Henry KD, Rosemond C, Eckert LB. Effect of number of home exercises on compliance and performance in adults over 65 years of age. Physical Therapy 1999; 79: 270-277

[21] Medina-Mirapeix F, Escolar-Reina P, Gascon-Canovas J] et al. Predictive factors of adherence to frequency and duration components in home exercise programs for neck and low back pain: an observational study. BMC Musculoskelet Disord 2009; 10: 155

[22] Eckard T, Lopez ], Kaus A et al. Home exercise program compliance of service members in the deployed environment: an observational cohort study. Military Medicine 2015; 180: 186-191

[23] Brewer BW, Van Raalte JL, Cornelius AE et al. Psychological factors, rehabilitation adherence, and rehabilitation outcome after anterior cruciate ligament reconstruction. Rehabil Psychol 2000; 45: 20-37

[24] Beinart NA, Goodchild CE, Weinman JA et al. Individual and intervention-related factors associated with adherence to home exercise in chronic low back pain: a systematic review. Spine J 2013; 13 : 1940-1950

[25] Chen C-Y, Neufeld PS, Feely CA et al. Factors influencing compliance with home exercise programs among patients with upper-extremity impairment. American Journal of Occupational Therapy 1999; 53: $171-180$
[26] McAuley E, Courneya KS, Rudolph DL et al. Enhancing exercise adherence in middle-aged males and females. Preventive Medicine 1994; 23: 498-506

[27] Schoo AMM, Morris ME, Bui QM. Predictors of home exercise adherence in older people with osteoarthritis. Physiotherapy Canada 2005; 57: 179-187

[28] Brewer BW, Cornelius AE, Van Raalte JL et al. Predictors of adherence to home rehabilitation exercises following anterior cruciate ligament reconstruction. Rehabil Psychol 2013; 58: 64-72

[29] Garay-Sevilla ME, Nava LE, Malacara JM et al. Adherence to treatment and social support in patients with non-insulin dependent diabetes mellitus. Journal of Diabetes and its Complications 1995; 9: 81-86

[30] Jeragh-Alhaddad FB, Waheedi M, Barber ND et al. Barriers to medication taking among Kuwaiti patients with type 2 diabetes: a qualitative study. Patient Preference and Adherence 2015; 9: 1491-1503

[31] Osterberg L, Blaschke T. Adherence to medication. New England Journal of Medicine 2005; 353: 487-497

[32] Schäfer B. Barrieren und Förderfaktoren der eigenständigen Durchführung von Übungen. physioscience. 2017; 13: 17-24 